\title{
Arriostramiento De PARAPETOS EXISTENTES HECHOS DE ALBAÑILERÍA SIMPLE
}

\author{
Ángel Francisco San Bartolome-Ramos ${ }^{1}$, Reymundo Anderson Siancas-Chavarría ${ }^{2}$, \\ Alan Gabriel Manrique-Guillén ${ }^{2}$
}

${ }^{1}$ Docente del Departamento de Ingeniería. Correo electrónico: asanbar@pucp.edu.pe

2 Estudiante de la Facultad de Ciencias e Ingeniería

Pontificia Universidad Católica del Perú

Recibido: 13 de julio del 2013. Aprobado: 5 de septiembre del 2013.

Cómo citar este artículo: Á. F. San Bartolome-Ramos, R. A. Siancas-Chavarría y A. G. Manrique-Guillén, "Arriostramiento de parapetos existentes hechos de albañilería simple". Ingeniería Solidaria, Vol. 9, №. 16, pp. 25-38, Dic., 2013.

Resumen. En diversos terremotos ocurridos en el Perú ha podido apreciarse que los parapetos de albañilería simple (no reforzada) terminan volcándose, poniendo en riesgo a las personas que transitan por la zona o que escapan del mismo edificio. Ante este peligro y dada la existencia de una gran cantidad de parapetos sueltos en el Perú, es necesario tomar medidas preventivas, por lo que en este proyecto se buscó una técnica económica y sencilla de aplicar que permita arriostrarlos adecuadamente evitando su colapso. Esta técnica consistió en emplear pequeñas columnas en los bordes del parapeto, anclando químicamente su refuerzo vertical en la base. Se varió la disposición del refuerzo así como su forma de anclar. La técnica fue verificada mediante ensayos de simulación sísmica hechos en la mesa vibradora de la PUCP durante 2012 y 2013, donde se ensayaron en simultáneo a cuatro parapetos. Puesto que dos parapetos arriostrados soportaron la acción sísmica, hubo que ensayarlos estáticamente para así determinar su resistencia máxima. En síntesis, el parapeto de albañilería simple colapsó para cargas inferiores a las reglamentarias, mientras que los arriostrados soportaron cargas mayores.

Palabras clave: albañilería, arriostre, ensayo sísmico, parapeto, terremoto.

\section{Bracing of Existing Simple Masonry Parapets}

Abstract. Various earthquakes in Peru have shown that simple masonry (that is, not reinforced) parapets collapse, placing people in the area and those exiting the building at risk. Due to this risk, and the existence of many non-reinforced parapets in Peru, it is essential that preventative measures are taken. Accordingly, this project examines an economical and simple technique that can be used to adequately brace the parapets, thereby preventing them from collapsing. The technique consists of employing small columns at the edges of the parapet and chemically anchoring the vertical reinforcement to the base. Different positions and anchoring methods were tested. This technique was verified through seismic simulation tests on the PUCP's shake table in 2012 and 2013, testing four parapets simultaneously. Two braced parapets withstood the seismic action, and these then underwent static trials to determine their maximum resistance. In summary, the simple masonry parapet collapsed under loads below regulatory limits, while the braced parapets supported greater loads.

Keywords: masonry, bracing, seismic test, parapet, earthquake.

\section{ESCORAMENTO DE PARAPETOS EXISTENTES FEITOS DE ALVENARIA SIMPLES}

Resumo. Em diversos terremotos ocorridos no Peru, pôde-se observar que os parapeitos de alvenaria simples (não reforçada) terminam caindo, o que põe em risco as pessoas que transitam pela zona ou que fogem do próprio edifício. Ante esse perigo e dada a existência de uma grande quantidade de parapeitos soltos no Peru, é necessário tomar medidas preventivas, razão pela qual neste projeto se procurou uma técnica econômica e simples de aplicar que permita escorá-lo adequadamente e evitar seu colapso. Essa técnica consistiu em empregar pequenas colunas nos bordes do parapeito, que fixassem quimicamente seu reforço vertical na base. Variou-se a disposição do reforço, bem como sua forma de fixar. A técnica foi verificada mediante ensaios de simulação sísmica feitos na mesa vibradora da PUCP durante 2012 e 2013, onde se ensaiaram simultaneamente quatro parapeitos. Posto que dois parapeitos escorados suportaram a ação sísmica, foi necessário ensaiá-los estaticamente para assim determinar sua resistência máxima. Em síntese, o parapeito de alvenaria simples colapsou para cargas inferiores às regulamentares, enquanto os escorados suportaram cargas maiores.

Palavras-chave: alvenaria, escoramento, ensaio sísmico, parapeito, terremoto. 


\section{Introducción}

Se sabe que el Perú se encuentra ubicado en una zona altamente sísmica y que existen numerosas edificaciones cuyas fachadas presentan parapetos de albañilería que, al carecer de arriostres (figura 1), podrían volcarse ante cargas sísmicas perpendiculares a su plano, generando un grave peligro para las personas que circulan por las inmediaciones o aquellas que escapan del mismo edificio. Por eso, en este trabajo se analizaron tres tipos de arriostres económicos y sencillos de construir, pero que permitirían evitar el colapso de estos parapetos.
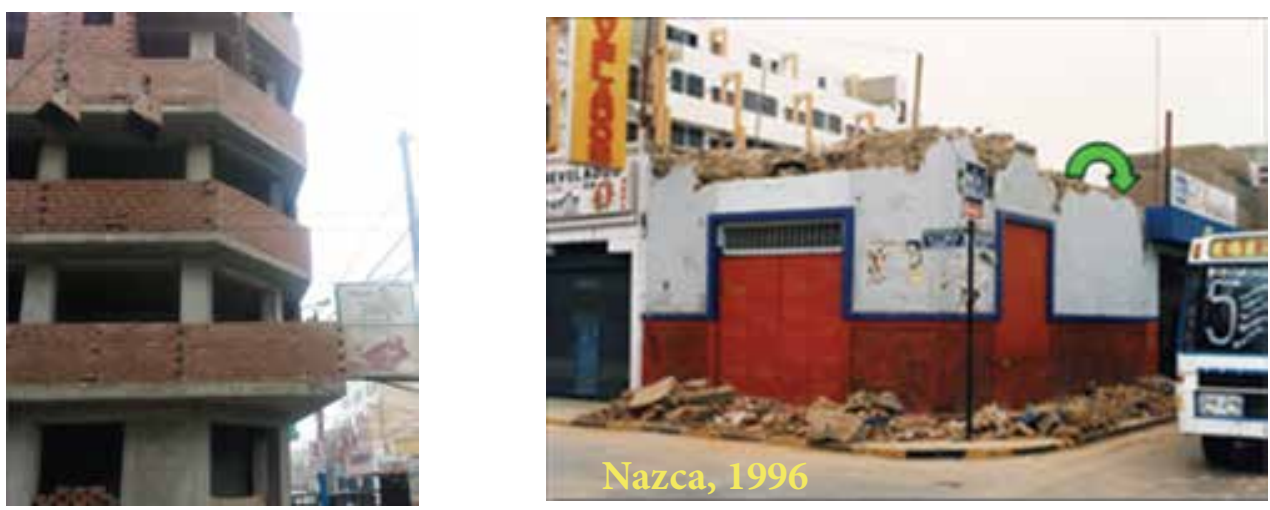

Figura 1. Parapeto de albañilería sin arriostrar y volcamiento Fuente: los autores

\section{Características de los especímenes}

\subsection{Características de los parapetos analizados}

Se analizaron cuatro parapetos de albañilería (figura 2), cada uno de longitud total $L=1,2 \mathrm{~m}$, altura $\mathrm{h}=1,0$ $\mathrm{m}$ y espesor $\mathrm{t}=12 \mathrm{~cm}$. Tres de estos parapetos fueron arriostrados con columnas de $12 \times 12 \mathrm{~cm}$, ubicadas en sus bordes, con distinta disposición y forma de anclaje del refuerzo vertical. En los tres parapetos arriostrados, se trató de simular el reforzamiento de un parapeto existente real hecho de albañilería simple, construyendo primeramente la albañilería, dejando dientes en sus extremos, para después instalar el refuerzo vertical y vaciar el concreto de las columnas. En un caso real habría que picar la albañilería en forma dentada en la zona donde irán las columnas.
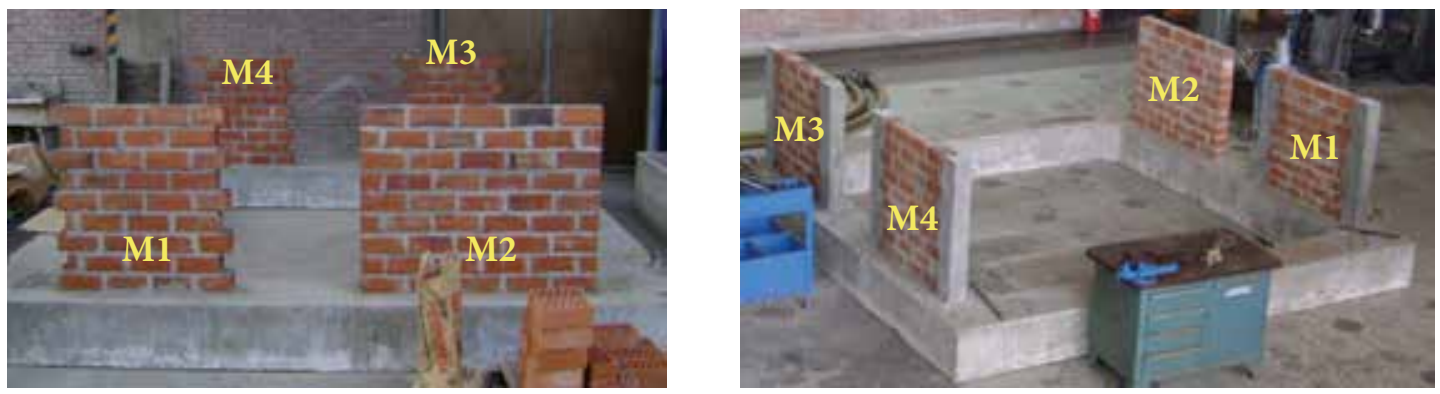

Figura 2. Parapetos analizados. Primero se construyó la albañilería sobre un anillo de concreto armado Fuente: los autores 
Las características de estos parapetos fueron:

- Se usaron ladrillos de arcilla del tipo King Kong con 18 huecos. Estas unidades son absorbentes de agua, por lo que para evitar que el mortero se endurezca por el agua succionada por los ladrillos hubo que regarlas durante media hora unas diez horas antes de asentarlas [1].

- El refuerzo de las columnas fue de acero corrugado Grado 60 (fy $=4.200 \mathrm{~kg} / \mathrm{cm} 2$ o $420 \mathrm{MPa}$ ).

- La dosificación del mortero fue 1:5 (una parte de cemento por cinco de arena gruesa).

- El concreto utilizado para las columnas tuvo una resistencia nominal f'c $=210 \mathrm{~kg} / \mathrm{cm} 2(21 \mathrm{MPa})$.

- Las juntas de mortero fueron de $1 \mathrm{~cm}$ de espesor y los parapetos no fueron tarrajeados.

- El parapeto denominado M2 fue el patrón de comparación ya que representa a los parapetos que carecen de arriostres (albañilería simple).

- El parapeto M1 fue arriostrado con dos columnas en sus extremos, reforzadas con una varilla de $8 \mathrm{~mm}$ de diámetro colocada en el centro de la columna y anclada químicamente con epóxico en una perfora- ción hecha en la cimentación (figura 3), de $1 \mathrm{~cm}$ de diámetro y una profundidad de $15 \mathrm{~cm}$.

- El parapeto M3 fue arriostrado con dos columnas en sus extremos, reforzadas con dos varillas de $1 / 4$ " de diámetro colocadas en el centro de la columna en la dirección del lado más débil. Estas varillas fueron amarradas con estribos de $1 / 4$ " con ganchos a $180^{\circ} \mathrm{y}$ anclaron químicamente con epóxico en un hueco hecho en la cimentación (figura 4), de $5 \times 10 \mathrm{~cm}$ y $10 \mathrm{~cm}$ de profundidad.

El parapeto M4 fue muy parecido a M3, excepto en el anclaje del refuerzo vertical. En este caso, las dos varillas de 1/4" de diámetro anclaron químicamente con epóxico en dos perforaciones hechas en la cimentación (figura 5), de $1 \mathrm{~cm}$ de diámetro y $15 \mathrm{~cm}$ de profundidad.

La mano de obra fue única en la construcción de los cuatro parapetos. Las perforaciones y el hueco en la cimentación se hicieron con un taladro eléctrico.

$\mathrm{El}$ anillo de cimentación de concreto armado fue de forma cuadrada, de 3,6 x 3,6 m y tuvo una sección transversal de $0,3 \times 0,3 \mathrm{~m}$.
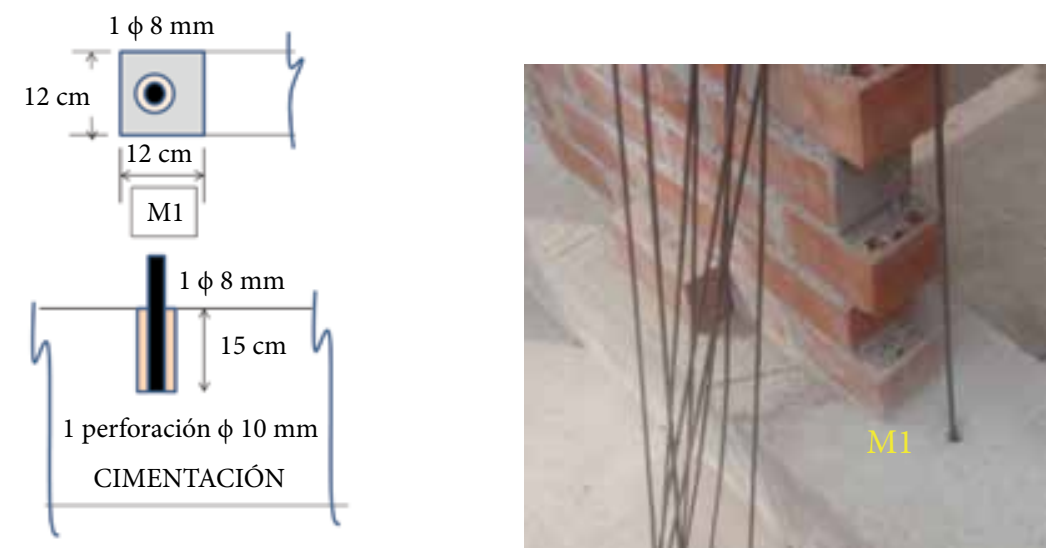

Figura 3. Refuerzo y anclaje en columnas del parapeto M1 Fuente: los autores 

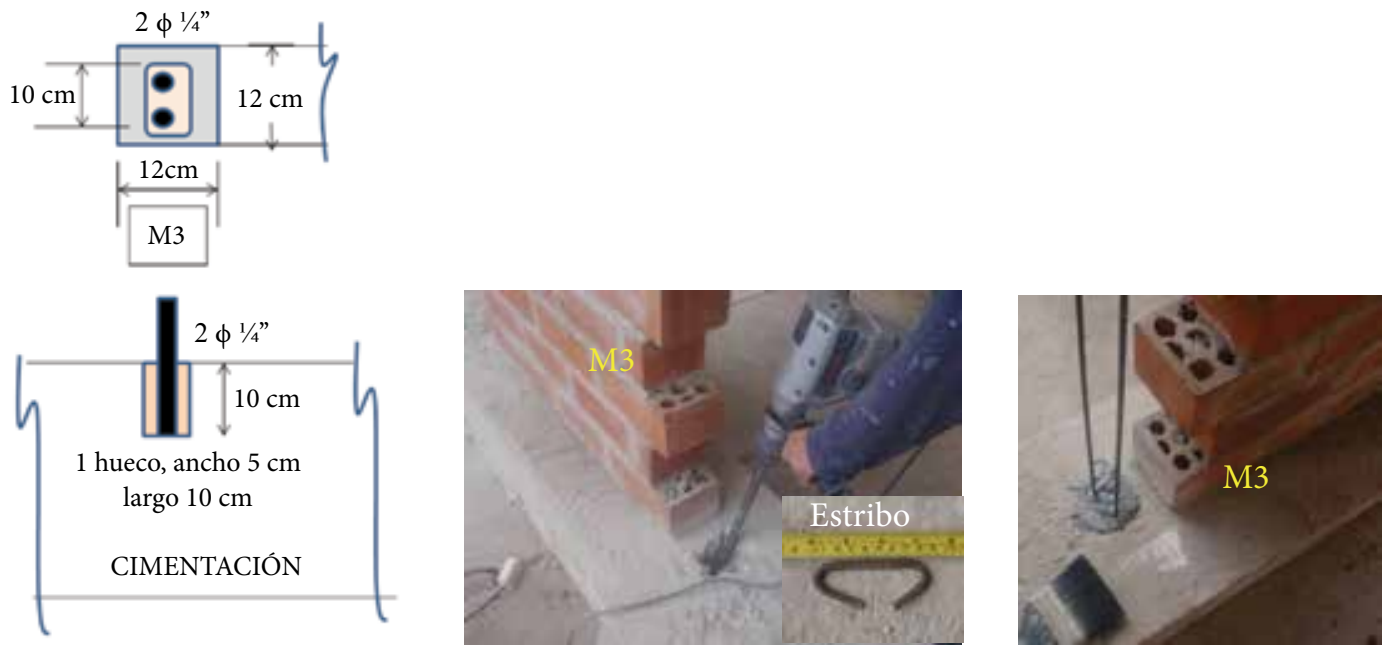

Figura 4. Refuerzo y anclaje en columnas del parapeto M3

Fuente: los autores
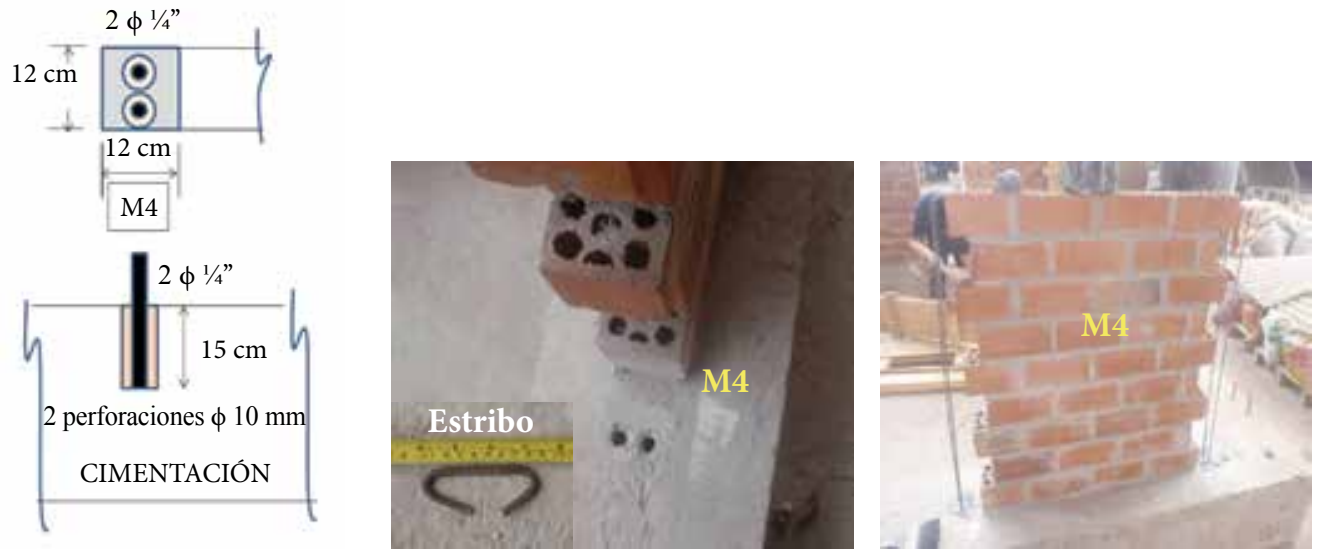

Figura 5. Refuerzo y anclaje en columnas del parapeto M4

Fuente: los autores

\subsection{Epóxico (Euco \#452 Gel)}

Se usó epóxico Euco 452 Gel (figura 6, [2]) para anclar químicamente las varillas de refuerzo vertical de las columnas en el anillo de cimentación. Este epóxico es un adhesivo para elementos endurecidos y de distinta calidad, además es insensible a la humedad y puede adherir en superficies secas o mojadas, previa limpieza con aire comprimido de las perforaciones hechas en la cimentación.
Se siguieron las especificaciones del fabricante, que textualmente indica lo siguiente:

[...] para la instalación de anclajes, inserte la punta mezcladora estática y llene el hoyo desde abajo, lentamente retirando la punta a medida que se dosifica el epóxico hasta que el hoyo esté lleno casi hasta la mitad. Inserte el anclaje con un movimiento lento y giratorio para garantizar contacto completo con el epóxico sin vacíos de aire. Estabilice el anclaje hasta que se haya curado el epóxico [2]. 


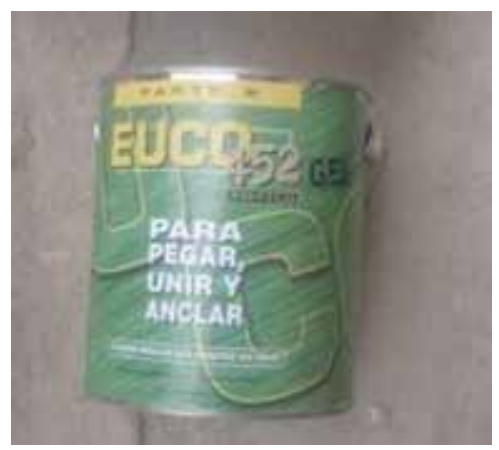

Figura 6. Epóxico Euco 452 Gel y anclaje químico de barras Fuente: los autores

\section{Evaluación sísmica de los parapetos}

\subsection{Carga sísmica reglamentaria perpendicular al parapeto}

De acuerdo con el Ministerio de Vivienda, Construcción y Saneamiento y Servicio Nacional de Capacitación para la Industria de la Construcción (SENCICO) [1], la carga sísmica elástica perpendicular al plano del parapeto es:

$$
\mathrm{w}=0,8 \mathrm{Z} \mathrm{UC1} \text { y e }
$$

donde los parámetros $\mathrm{Z}, \mathrm{U}$ y C1 se obtienen de [3]:

Z: factor de zona sísmica, para la costa peruana $\mathrm{Z}$ $=0,4$

$\mathrm{U}$ : factor de importancia, para viviendas y oficinas $\mathrm{U}=1,0$

C1: coeficiente sísmico, para elementos que puedan precipitarse fuera de la edificación $\mathrm{C} 1=$ 1,3

$\gamma: \quad$ peso volumétrico de la albañilería, $\gamma=1.800$ $\mathrm{kg} / \mathrm{m} 3$

e: espesor bruto del muro (incluyendo tarrajeos), $\mathrm{e}=0,12 \mathrm{~m}$

De esta manera, la carga perpendicular a la albañilería es

$$
\begin{gathered}
\mathrm{w}=0,8 \times 0,4 \times 1 \times 1,3 \times 1.800 \times 0,12= \\
90 \mathrm{~kg} / \mathrm{m} 2(0,9 \mathrm{kPa})
\end{gathered}
$$

En tanto que la carga última se logra amplificando a "w" por 1,25: wu = 1,25 x $90=112 \mathrm{~kg} / \mathrm{m} 2(1,12 \mathrm{kPa})$.

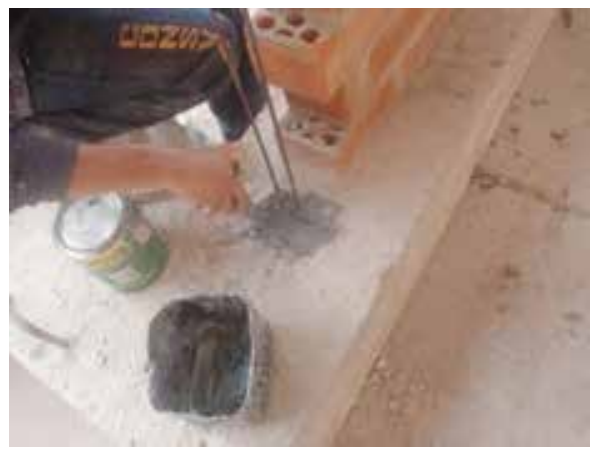

\subsection{Evaluación del parapeto no arriostrado (M2)}

Este parapeto se comporta como un elemento en voladizo, donde el momento flector en la base es:

$$
M=(w L h) h / 2=\frac{w L h^{2}}{2}
$$

De esta manera: $\mathrm{M}=90 \times 1,2 \times 1,02 / 2=54 \mathrm{~kg}-\mathrm{m}=$

$$
5.400 \mathrm{~kg}-\mathrm{cm} \text {. }
$$

El esfuerzo normal de tracción originado por " $M$ " en el borde inferior del parapeto "M2" es:

$$
\sigma=\frac{M y}{I}=\frac{M t / 2}{\frac{L t^{3}}{12}}=\frac{6 M}{L t^{2}}
$$

$$
\begin{aligned}
& \text { Con lo cual } \sigma=6 \times 5.400 /\left(120 \times 12^{2}\right)=1,9 \mathrm{~kg} / \\
& \mathrm{cm}^{2}(0,19 \mathrm{MPa})
\end{aligned}
$$

Como para la carga elástica reglamentaria, $\sigma=1,9$ $\mathrm{kg} / \mathrm{cm}^{2}(0,19 \mathrm{MPa})$ supera al esfuerzo admisible permitido por el Ministerio de Vivienda, Construcción y Saneamiento y SENCICo [1]: 1,5 kg/cm2 (0,15 MPa), el parapeto M2 necesita ser arriostrado.

\subsection{Evaluación elástica de los arriostres y de la albañilería en los parapetos M1, M3 y M4}

Una vez que se añaden las columnas, la albañilería se comporta como una losa apoyada en tres bordes (dos columnas y la base), debiéndose verificar que el borde libre superior de la albañilería no se agriete por flexión, al igual que la base de las columnas. Para ello se asume que la distribución de cargas sísmicas responde a la denominada regla del sobre (figura 7). 

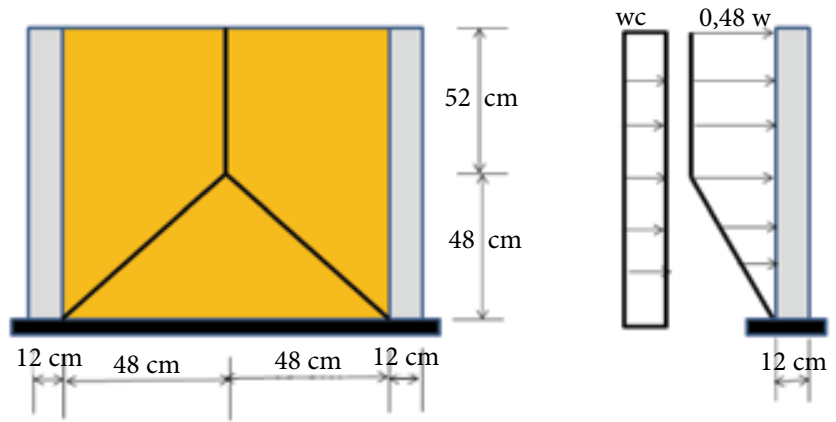

Figura 7. Esquema de la distribución de cargas sísmicas en la albañilería arriostrada Fuente: los autores

El momento flector actuante en la zona central superior de la albañilería (borde libre, zona más crítica a flexión), está dado por la norma E.070 [1] a través de la expresión:

$$
M s=m w a^{2}
$$

Donde:

$\mathrm{m}$ : coeficiente de momento adimensional, indicado en la tabla 12 de [1],

$\mathrm{m}=0,112$ (caso de tres bordes arriostrados, con $\mathrm{h}$ $/ \mathrm{a}=1 / 0,96=1,04)$

a: dimensión crítica del paño de albañilería, o longitud del borde libre, $a=0,96 \mathrm{~m}$

Con lo cual: $\mathrm{Ms}=0,112 \times 90 \times 0,962=9,3 \mathrm{~kg}-\mathrm{m} / \mathrm{m}$

El esfuerzo de tracción por flexión producido por "Ms" está dado por:

$$
\sigma=\frac{6 M s}{t^{2}}
$$

Por lo que: $\sigma=6 \times 9,3 /\left(0,12^{2}\right)=3.875 \mathrm{~kg} / \mathrm{m} 2=$ $0,39 \mathrm{~kg} / \mathrm{cm} 2(0,039 \mathrm{MPa})$. Puesto que " $\sigma$ " es menor que la resistencia admisible a tracción por flexión de la albañilería simple $\left(1,5 \mathrm{~kg} / \mathrm{cm}^{2}\right.$ o $\left.0,15 \mathrm{MPa},[1]\right)$, la albañilería no se agrietará en su zona superior, hasta que se alcance una carga mayor que wfa $=90(1,5 / 0,39)=346$ $\mathrm{kg} / \mathrm{m}^{2}(3,46 \mathrm{kPa})$.

La carga en la columna proveniente de la albañilería es trapezoidal, mientras que la carga producto de su peso propio es uniforme:

$$
\mathrm{wc}=8,0 \mathrm{Z} \mathrm{U} \mathrm{C1} \mathrm{yc} \mathrm{Ac}
$$

De la cual se obtiene:

$\mathrm{wC}=0,8 \times 0,4 \times 1,0 \times 1,3 \times 2.400 \times 0,12 \times 0,12=14,4 \mathrm{~kg} / \mathrm{m}$,

con lo cual, el momento flector en la base de la columna es:

$\mathrm{Mc}=14,4 \times 1,0^{2} / 2+0,48 \times 90 \times 0,52(0,52 / 2+0,48)+1 / 2 \times$ $0,48 \times 90 \times 0,48 \times(2 \times 0,48 / 3)=27,14 \mathrm{~kg}-\mathrm{m}(271,4 \mathrm{~N}-\mathrm{m})$

El esfuerzo de tracción por flexión en la base de la columna es:

$$
\sigma=\mathrm{Mc} y / \mathrm{I}=2.714 \times 6 /\left(12 \times 12^{3} / 12\right)=9,4 \mathrm{~kg} / \mathrm{cm}^{2}
$$

Este valor es inferior a la resistencia a tracción por flexión del concreto

$$
\left(2 \sqrt{ } f^{\prime} \mathrm{c}=2 \sqrt{2} 210=29 \mathrm{~kg} / \mathrm{cm}^{2} \text { o } 2,9 \mathrm{MPa},[4]\right),
$$

por lo que el concreto se fisuraría para una carga $\mathrm{wfc}=$ $90(29 / 9,4)=278 \mathrm{~kg} / \mathrm{m}^{2}(2,78 \mathrm{kPa})$. Puesto que "wfc" es menor que "wfa", primero se agrietaría la base de la columna.

\subsection{Capacidad resistente de los parapetos arriostrados M1, M3 y M4}

Una vez que fisura la base de las columnas en tracción por flexión, se pierde el comportamiento indicado en el acápite 3.3 y los parapetos se comportan como elementos en voladizo, donde la capacidad resistente la dan los momentos flectores proporcionados por el refuerzo vertical. La magnitud de la carga resistente " $w$ " dependerá del anclaje del refuerzo, del área de acero colocada y de la posibilidad de que este ingrese en su zona de endurecimiento. Se asumirá que el refuerzo vertical fluye y se adoptará la siguiente nomenclatura (figura 8): 

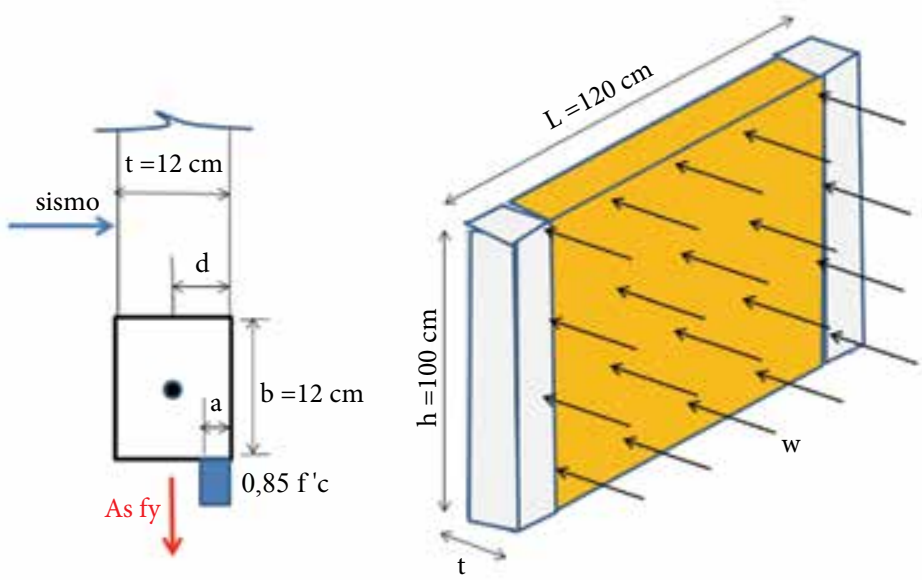

Figura 8. Esquema de cuerpo libre en la base de una columna Fuente: los autores

$\mathrm{d}=$ distancia entre el eje de la varilla traccionada y el borde extremo comprimido

$\mathrm{b}=12 \mathrm{~cm}=$ ancho de la columna

$\mathrm{f}^{\prime} \mathrm{c}=210 \mathrm{~kg} / \mathrm{cm}^{2}(21 \mathrm{MPa})$, resistencia a compresión del concreto

$\mathrm{fy}=4.200 \mathrm{~kg} / \mathrm{cm}^{2}(420 \mathrm{MPa})$, esfuerzo de fluencia del refuerzo vertical

As =área del refuerzo vertical

\subsubsection{Caso 1: M1, refuerzo 1 ф $8 \mathrm{~mm}$ (Figura 3)}

$\mathrm{d}=6,0 \mathrm{~cm}$

As $=0,5 \mathrm{~cm}^{2}$

Por equilibrio de fuerzas verticales se obtiene "a":

As f y $=0,85 f^{\prime}$ c a b

Reemplazando valores: $0,5 \times 4.200=0,85 \times 210 \times$

a $\mathrm{x} 12 \rightarrow \mathrm{a}=0,98 \mathrm{~cm}$

Con lo cual, el momento nominal resistente es:

$$
M n=A s f y\left(d-\frac{a}{2}\right)
$$

$\mathrm{Mn}=0,5 \times 4.200(6-0,98 / 2)=11.571 \mathrm{~kg}-\mathrm{cm}=116$ kg-m (1160 N-m).

Luego, la carga que produce la fluencia del refuerzo se obtiene por equilibrio de momentos flectores en la base del parapeto:

$$
\begin{gathered}
2 M n=\frac{w L h^{2}}{2} \\
w=\frac{4 M n}{L h^{2}}
\end{gathered}
$$

De esta manera:

$\mathrm{w}=4 \times 116 /\left(1,2 \times 1,0^{2}\right)=387 \mathrm{~kg} / \mathrm{m}^{2}(3,87 \mathrm{KPa})$

Este valor supera a la carga última de diseño (wu $=112 \mathrm{~kg} / \mathrm{m}^{2} \mathrm{o} 1,12 \mathrm{KPa}$, acápite 3.1 ), por tanto, el refuerzo es excesivo.

\subsubsection{Caso 2: M3 y M4, refuerzo $2 \phi 1 / 4$ " (figuras 4 y5)}

Siguiéndose un procedimiento similar al Caso 1 (acápite 3.4.1), se tiene:

$$
\begin{aligned}
& \mathrm{d}=9 \mathrm{~cm} \\
& \text { As }=0,32 \mathrm{~cm}^{2}
\end{aligned}
$$

Por equilibrio de fuerzas verticales se obtiene “a $=0,63 \mathrm{~cm}$ ".

Con lo cual, el momento nominal resistente es:

$$
\begin{gathered}
\mathrm{Mn}=0,32 \times 4.200(9-0,63 / 2)=11.672 \mathrm{~kg}-\mathrm{cm}=117 \\
\mathrm{~kg}-\mathrm{m}(1170 \mathrm{~N}-\mathrm{m})
\end{gathered}
$$

Luego, la carga que produce la fluencia del refuerzo se obtiene por equilibrio de momentos en la base $\mathrm{w}$ $=4 \times 117 /(1,2 \times 1,02)=390 \mathrm{~kg} / \mathrm{m}^{2}(3,9 \mathrm{KPa})$. Este valor supera a la carga última de diseño $\left(\mathrm{w}_{\mathrm{u}}=112 \mathrm{~kg} / \mathrm{m}^{2} \mathrm{o}\right.$ $1,12 \mathrm{KPa}$, acápite 3.1), por tanto, el refuerzo es excesivo.

\subsubsection{Predicción de resultados}

El parapeto M2 requiere de arriostramiento, en caso contrario colapsaría. En cuanto a los tres parapetos arriostrados, cabe señalar que las resistencias a flexión para las dos disposiciones de refuerzo consideradas son muy parecidas, por lo que M1, M3 y M4 deberían tener igual comportamiento sísmico, salvo que existan problemas de anclaje del refuerzo vertical. Por otro lado, puesto que existe sobre 
resistencia a flexión, se espera que en el ensayo la carga sísmica pueda superar a la carga última de diseño $\left(\mathrm{w}_{\mathrm{u}}=\right.$ $112 \mathrm{~kg} / \mathrm{m}^{2} \mathrm{o} 1.12 \mathrm{KPa}$ ), así como a la carga que produce la fisura en tracción por flexión en la base de la columna (278 $\mathrm{kg} / \mathrm{m}^{2}$ o $2,78 \mathrm{KPa}$ ), sin que se produzca fisuras de tracción por flexión en el borde central superior de la albañilería, ya que su comportamiento cambiaría de una losa arriostrada en tres bordes (figura 7) a un muro en voladizo (figura 8). Estos parapetos arriostrados podrían alcanzar una resistencia del orden de $390 \mathrm{~kg} / \mathrm{m}^{2}(3,9 \mathrm{KPa})$, asociada a la fluencia del refuerzo vertical, e incluso podría ser mayor ( $50 \%$ más) si es que el refuerzo vertical ingresara en su zona de endurecimiento y no hubiese falla por anclaje.

\section{Ensayo de simulación sísmica}

Los cuatro parapetos fueron sometidos en simultáneo a la componente "L" del terremoto ocurrido el 31 de mayo de 1970 en Chimbote. Esta señal tiene 30 segundos de duración y un contenido de frecuencias predominantes comprendida entre 2,5 y 3,5 Hz. Se aplicaron tres fases de aceleración (Ao) creciente, tratando de simular sismos leves, moderados y severos (tabla 1). En cada parapeto se utilizaron dos sensores (figura 9): un acelerómetro localizado en el centro del parapeto (Ai) y un LVDT (Di), o Transformador Diferencial de Variación Lineal, ubicado en el borde central superior del parapeto.
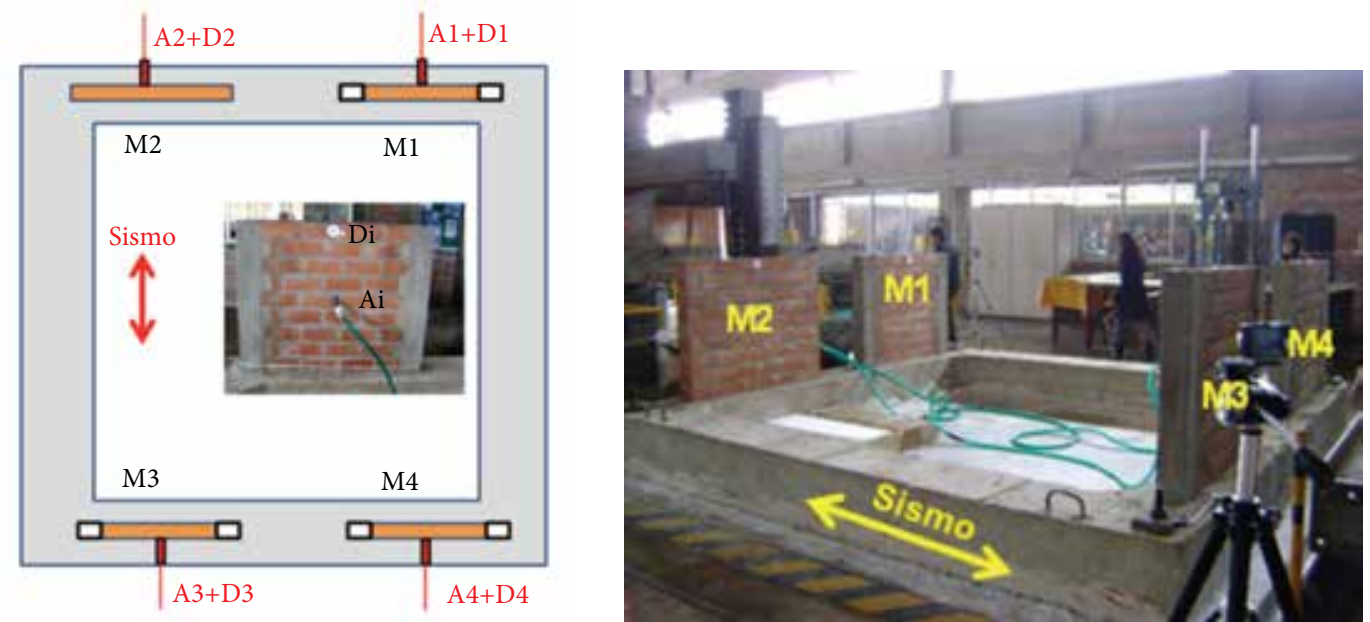

Figura 9. Instrumentación

Fuente: los autores

Tabla 1. Fases del ensayo sísmico

\begin{tabular}{|l|l|l|l|r|}
\hline \multirow{2}{*}{ Fase } & \multicolumn{2}{|c|}{$\begin{array}{c}\text { Aceleración máxima de la mesa } \\
\text { Ao }(\mathrm{g})\end{array}$} & \multicolumn{2}{c|}{$\begin{array}{c}\text { Desplazamiento máximo de la mesa } \\
\text { Do (mm) }\end{array}$} \\
\hline 1 & 0,49 & $-0,64$ & $-45,87$ & 60,70 \\
\hline 2 & 0,81 & $-1,05$ & $-74,72$ & 100,05 \\
\hline 3 & 1,19 & $-1,47$ & $-100,17$ & 133,59 \\
\hline
\end{tabular}

Fuente: elaboración propia

\subsection{Procesamiento de resultados}

Los desplazamientos relativos a la mesa (deformación por flexión), se obtuvieron restando al desplazamiento absoluto captado por el LVDT el desplazamiento de la plataforma (Do): di = Di - Do.

La carga sísmica perpendicular al plano del parapeto $(\mathrm{Fi})$ se obtuvo multiplicando el peso del parapeto (Pi) por la aceleración en fracción de "g" medida en su centroide (Ai): Fi = Pi Ai. Luego esta fuerza se dividió entre el área del parapeto, para así obtener la fuerza dis- tribuida por unidad de área: wi $=\mathrm{Fi} /(\mathrm{L} \mathrm{h})=\mathrm{Fi} /(1,2 \mathrm{x}$ $\left.1 \mathrm{~m}^{2}\right)=\mathrm{Pi} \mathrm{Ai} / 1,2$. Los pesos de los parapetos fueron:

$\mathrm{P}(\mathrm{M} 2)=\gamma \mathrm{a} \mathrm{L} \mathrm{h} \mathrm{t}=1.800 \times 1,2 \times 1,0 \times 0,12=259,2$ $\mathrm{kg}(2.592 \mathrm{~N})$

$\mathrm{P}(\mathrm{M} 1, \mathrm{M} 3$ y M4) $=2 \gamma \mathrm{cAch}+\gamma \mathrm{aB} \mathrm{h} \mathrm{t}=$ $2 \times 2.400 \times 0,12 \times 0,12 \times 1,0+1.800 \times 0,96 \times 1,0 \times 0,12$ $=276,5 \mathrm{~kg}(2.765 \mathrm{~N})$.

Para obtener el instante en el cual fallaba un parapeto, se observó el video grabado en tiempo real y la historia 


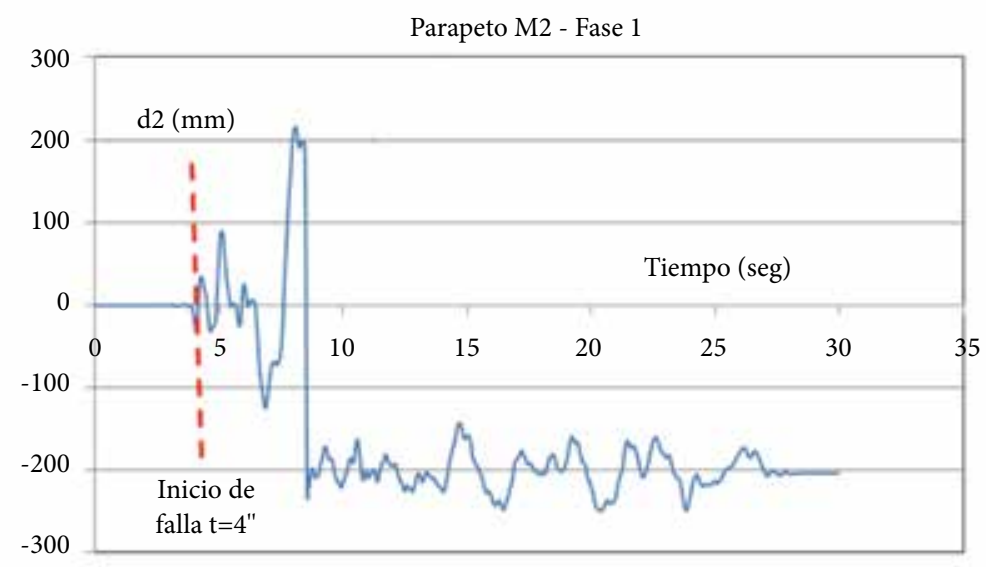

Figura 10. Historia de desplazamiento de M2 (no arriostrado) Fase 1

Fuente: los autores

de desplazamientos, registrándose el tiempo para el cual el desplazamiento se incrementaba fuertemente (figura 10); los datos para el tiempo restante se consideraron nulos.

\subsection{Comportamiento cualitativo de los parapetos}

El parapeto M2 (no arriostrado) volcó durante la fase 1 del ensayo (figura 11). Durante la fase 2, el parapeto

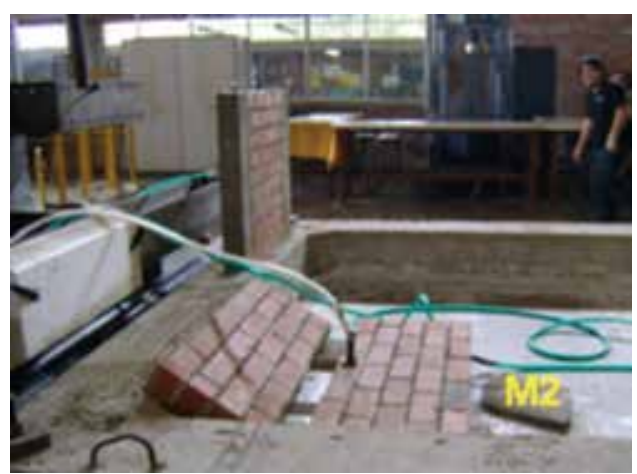

Figura 11. Parapeto M2 - Fase 1

Fuente: los autores

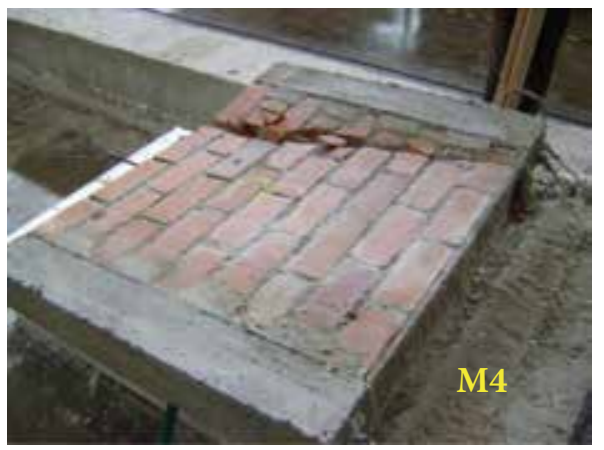

Figura 13. Parapeto M4-Fase 3. Falla por anclaje del refuerzo vertical Fuente: los autores
M4 se agrietó por flexión en su base (figura 12), para después volcarse durante la fase 3 (figura 13); la falla en M4 se produjo por anclaje del refuerzo vertical. Los parapetos que quedaron sin daños después de la fase 3 fueron: M1 y M3.

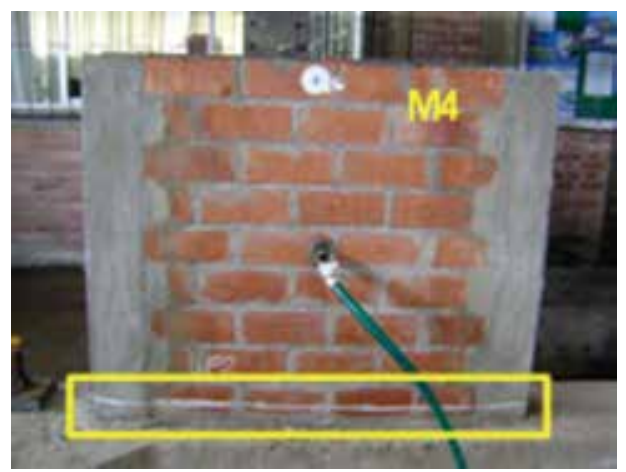

Figura 12. M4-Fase 2. Grieta en la base Fuente: los autores

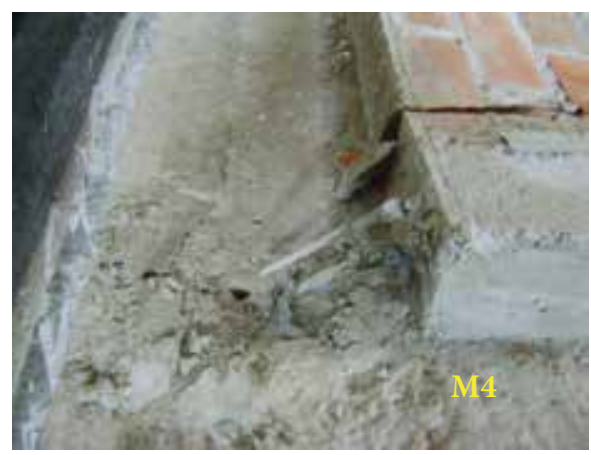




\subsection{Comportamiento cuantitativo de los parapetos}

Las gráficas w-d (figuras 14 a 16) han sido hechas a la misma escala con fines comparativos. En la fase 1
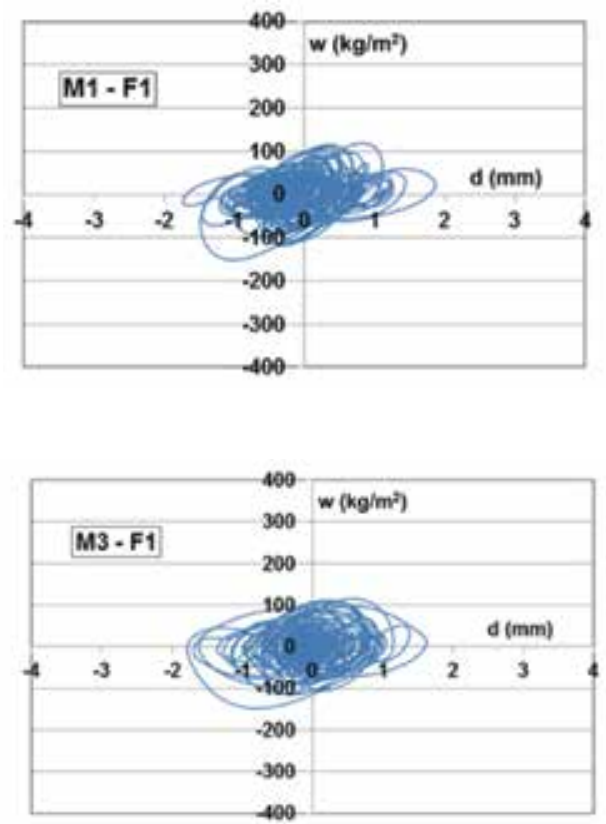

(figura14), se nota que los parapetos arriostrados (M1, M3 y M4) tienen prácticamente la misma respuesta, mientras que en M2 el desplazamiento creció fuertemente para después volcarse.
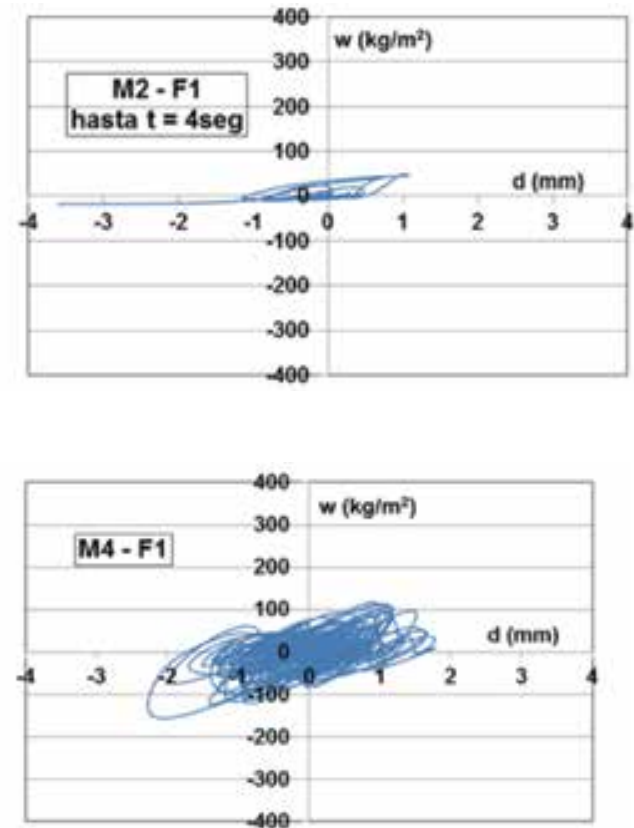

Figura 14. Fase 1. Gráficas w-d en M1 a M4. En esta fase colapsó M2 (no arriostrado)

Fuente: los autores

En la fase 2 (figura 15), los parapetos M1 y M3 respondieron de manera similar, mientras que en M4 se inició la falla en su base, incrementándose fuertemente el desplazamiento, para la fase 3 se retiró el LVDT D4.
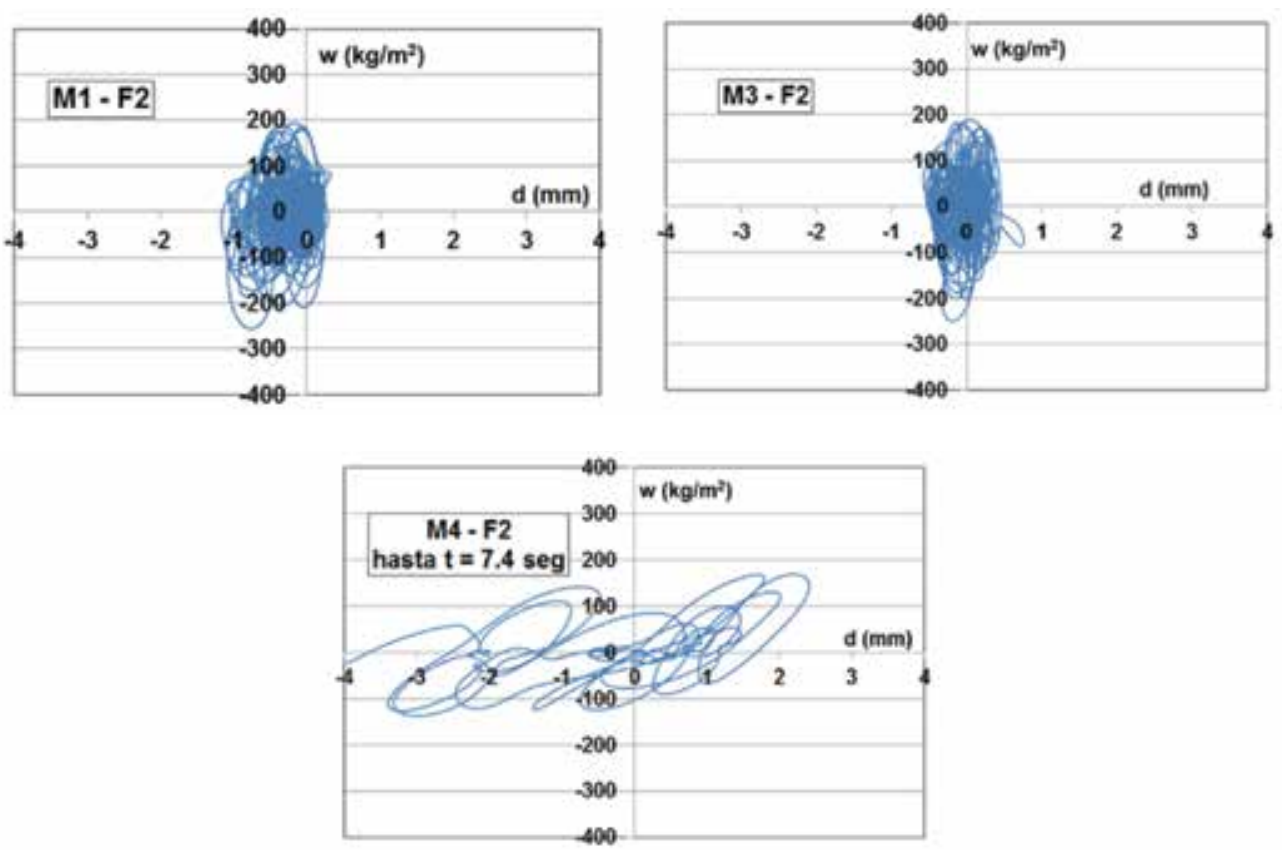

Figura 15. Fase 2. Gráficas w-d en M1, M3 y M4. En esta fase se formó una grieta en la base de M4 (figura 12)

Fuente: los autores 
En la fase 3 (figura16), M1 y M3 respondieron de manera similar, mientras que M4 se volcó.
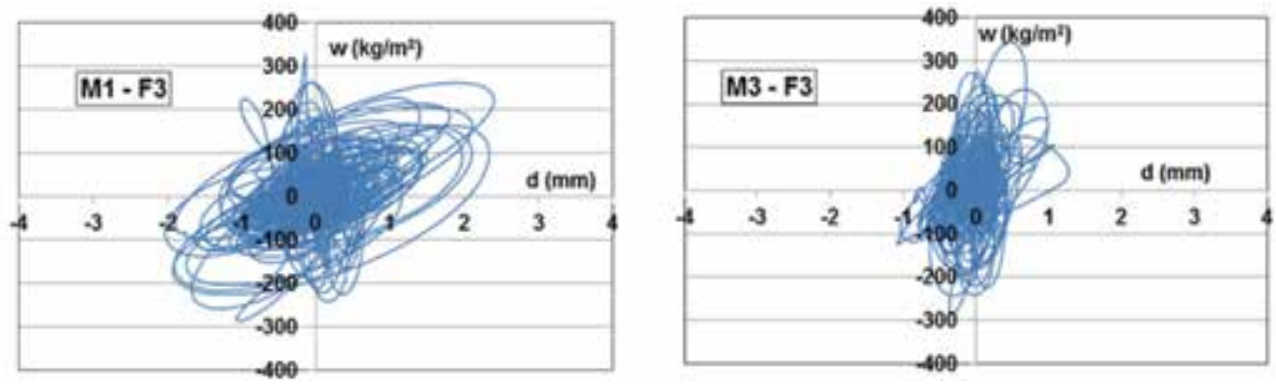

Figura 16. Fase 3. Gráficas w-d en M1 y M3. M4 se volcó en esta fase (figura 13)

Fuente: los autores

En la tabla 2 aparecen sólo los valores máximos de las cargas "w". Se ha preferido no tabular los desplazamientos porque estos son pequeños y se usaron LVDT de $200 \mathrm{~mm}$ de capacidad, lo que podría producir errores de precisión. Más bien la envolvente de cargas máximas (figura 17) fue obtenida graficando los valores de la tabla 2: "w" versus la aceleración máxima en la base "Ao".

Tabla 2. Cargas máximas “w” en $\mathrm{kg} / \mathrm{m}^{2}$, entre paréntesis en $\mathrm{KPa}$

\begin{tabular}{|l|l|l|l|l|}
\hline \multicolumn{1}{|c|}{ Fase } & \multicolumn{1}{|c|}{ M1 } & \multicolumn{1}{c|}{ M2 } & \multicolumn{1}{c|}{ M3 } & \multicolumn{1}{c|}{ M4 } \\
\hline $1(\mathrm{Ao}=0,64 \mathrm{~g})$ & $151(1,51)$ & $48(0,48)$ & $148(1,48)$ & $157(1,57)$ \\
\hline $2(\mathrm{Ao}=1,05 \mathrm{~g})$ & $255(2,55)$ & --- & $249(2,49)$ & $167(1,67)$ \\
\hline $3(\mathrm{Ao}=1,47 \mathrm{~g})$ & $328(3,28)$ & --- & $343(3,43)$ & $-\cdots$ \\
\hline
\end{tabular}

Fuente: elaboración propia

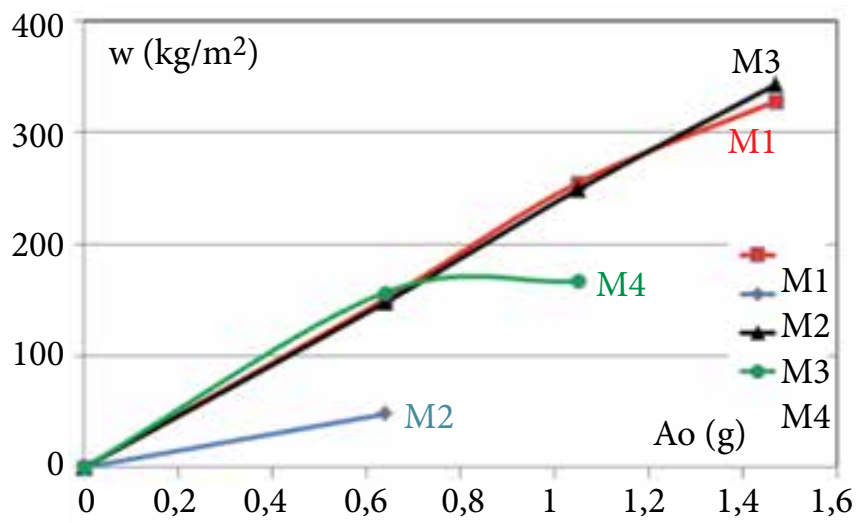

Figura 17. Envolvente de carga máxima versus aceleración en la base Ao Fuente: los autores

\section{Ensayos de carga estática}

Los parapetos M1 y M3 pudieron soportar el ensayo sísmico, por lo que para conocer su forma y su carga de falla fueron sometidos a un ensayo estático (figura18), consistente en aplicar con una gata hidráulica una carga concentrada (F) en el borde superior, medida con una celda de carga, mientras que el desplazamiento lateral (D) del punto central superior fue registrado con un LVDT.

Los dos parapetos fallaron volcándose por flexión a través de su base, observándose una falla por anclaje del refuerzo vertical en ambos casos (figura 19).

En la figura 20 se presenta la gráfica F-D de los parapetos M1 y M3. Allí se observa la mayor resistencia que tuvo M1. 


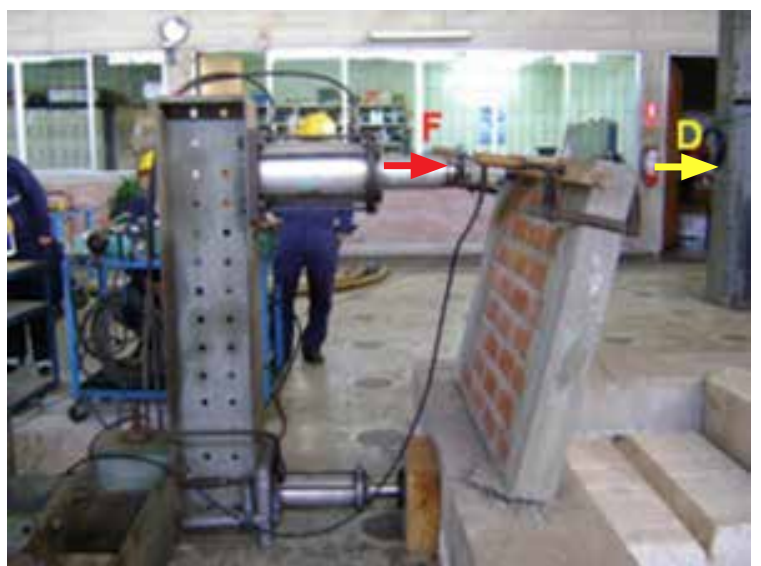

Figura 18. Ensayo estático

Fuente: los autores
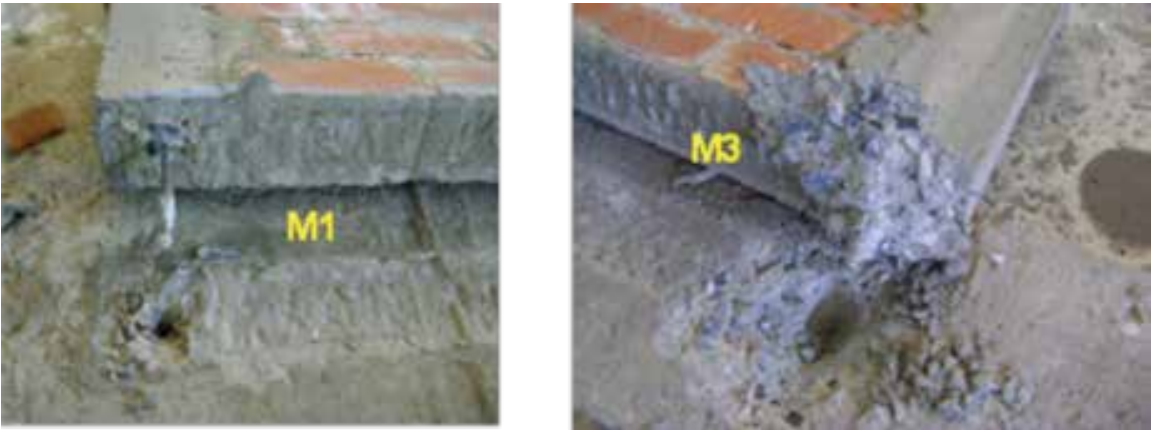

Figura 19. Ensayo estático. Falla por anclaje del refuerzo vertical en M1 y M3

Fuente: los autores

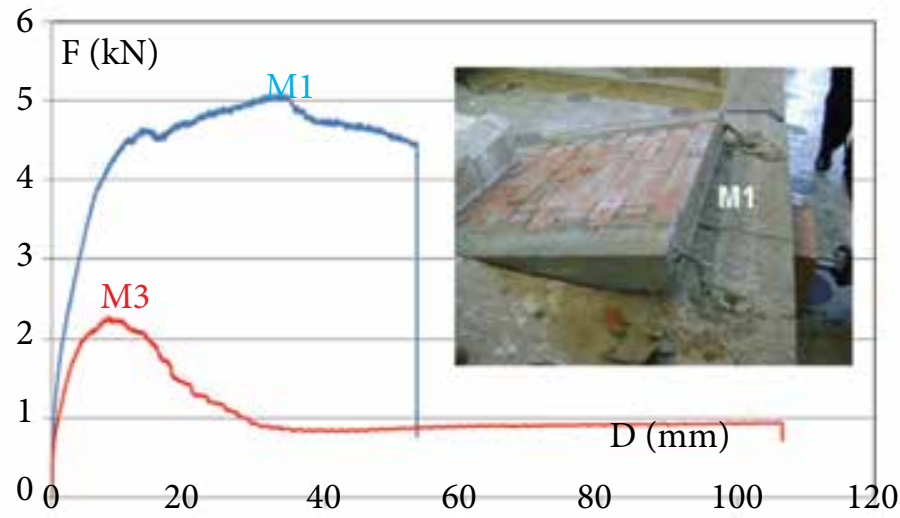

Figura 20. Ensayo estático. Gráfica F-D

Fuente: los autores

\subsection{Resistencia máxima y carga distribuida equivalente}

La carga aplicada en el ensayo estático (F) fue concentrada en el borde superior del parapeto, mientras que la carga sísmica real $(\mathrm{w})$ actúa en forma distribuida perpendicularmente al plano del parapeto (figura 8). Para buscar una equivalencia entre ambas cargas, y conociéndose que la falla final es por volcamiento, se igualaron los momentos flectores en la base producidos por estas cargas: 


$$
\begin{gathered}
M(F)=F h=M(w)=\frac{w L h h}{2}=\frac{w L h^{2}}{2} \\
w=\frac{2 F}{L h}
\end{gathered}
$$

Con lo cual, para el parapeto en análisis: $\mathrm{w}=2 \mathrm{~F}$ / $(1,2 \times 1,0)=1,67 \mathrm{~F}\left(\mathrm{en} \mathrm{kg} / \mathrm{m}^{2}\right)$. De esta manera, los re- sultados máximos del ensayo estático se muestran en la tabla 3.

Tabla 3. Resistencias en el ensayo estático

\begin{tabular}{|l|l|l|}
\hline & \multicolumn{1}{|c|}{ Parapeto M1 } & \multicolumn{1}{|c|}{ Parapeto M3 } \\
\hline $\mathrm{F}(\mathrm{kg})$ & $517(5.170 \mathrm{~N})$ & $232(2.320 \mathrm{~N})$ \\
\hline $\mathrm{w}\left(\mathrm{kg} / \mathrm{m}^{2}\right)=1,67 \mathrm{~F}$ & $864(8,64 \mathrm{KPa})$ & $387(3,87 \mathrm{KPa})$ \\
\hline
\end{tabular}

Fuente: elaboración propia

\section{Comparación de resistencias máximas}

Para efectos comparativos, debe tenerse en cuenta que la carga última de diseño reglamentario es $\mathrm{w}_{\mathrm{u}}=112 \mathrm{~kg} /$ $\mathrm{m}^{2}(1,12 \mathrm{KPa}$, acápite 3.1$)$, y que los valores teóricos de resistencia (acápite 3.4) fueron obtenidos suponiendo que el refuerzo vertical fluye. Sin embargo, para grandes desplazamientos, podría ingresar en su zona de endurecimiento amplificándose la resistencia en un factor de 1,5. En la tabla 4 aparecen las resistencias teóricas y experimentales para los cuatro parapetos.

Tabla 4. Resistencias máximas “ $w$ ” en kg/m², entre paréntesis en $\mathrm{KPa}$

\begin{tabular}{|l|l|l|l|l|}
\hline \multicolumn{1}{|c|}{ Parapeto } & \multicolumn{1}{c|}{ M1 } & \multicolumn{1}{c|}{ M2 } & \multicolumn{1}{c|}{ M3 } & \multicolumn{1}{c|}{ M4 } \\
\hline Teoría (fluencia) & $387(3,87)$ & No reforzado & $390(3,90)$ & $390(3,90)$ \\
\hline Teoría (endurecimiento) & $581(5,81)$ & No reforzado & $585(5,85)$ & $585(5,85)$ \\
\hline Ensayo sísmico & $328($ Fase 3) & 48 (Fase 1) & $343($ Fase 3) & 167 (Fase 2) \\
\hline Ensayo estático & $864(8,64)$ & -- & $387(3,87)$ & -- \\
\hline
\end{tabular}

Fuente: elaboración propia

De la tabla 4 se pueden extraer los siguientes comentarios:

- La resistencia de la albañilería no arriostrada (M2) es mínima e inferior a la carga reglamentaria de diseño, por lo que es imprescindible arriostrarla.

- M3 y M4 tuvieron el mismo refuerzo vertical; sin embargo, M4 falló antes por anclaje, aunque para una carga mayor que la de diseño reglamentario. M3 falló también por anclaje, pero para una carga 2,3 veces mayor que la de M4, sin alcanzar la resistencia a la fluencia.

- M1 fue el parapeto que tuvo mayor resistencia, incluso que la correspondiente al endurecimiento del refuerzo vertical, lo cual no se explica ya que el refuerzo falló por anclaje (figuras 19 y 20), salvo que el mecanismo final de resistencia sea distinto al considerado en el acápite 3.4 y deba considerarse el aporte de la albañilería.

\section{Conclusiones}

- La resistencia sísmica de los parapetos de albañilería simple (M2) es mínima e inferior a la carga reglamentaria de diseño; por tanto, es necesario arriostrarlos para prevenir su colapso.

- Evitar el colapso de los parapetos sueltos es muy importante porque su falla, incluso ante la acción de sismos leves o muy frecuentes, traería por consecuencia no sólo daños materiales, sino que además pondrían en alto riesgo a las personas que transitan por la zona o que escapan del mismo edificio durante el sismo. Los arriostres con base en columnas de concreto con refuerzo vertical anclado químicamente mediante resina epóxica fueron fáciles de construir, y el utilizado en el parapeto M1 (una sola varilla anclada en una perforación) fue el más senci- 
llo y el que proporcionó la mayor resistencia a carga sísmica perpendicular al plano del parapeto.

- Todos los parapetos arriostrados fallaron por anclaje del refuerzo vertical, con una carga mayor a la carga reglamentaria de diseño, pero menor al valor teórico de fluencia, excepto en M1, donde la resistencia sobrepasó incluso a la de endurecimiento del refuerzo vertical.

- Puesto que para un diseño teórico se trabaja con la carga de fluencia del refuerzo y dado que en este proyecto se obtuvieron resistencias muy aleatorias debido a la acción simultánea de tracción y flexión a que estuvieron sometidas las columnas, y también porque en ensayos de tracción directa se ha demostrado que el refuerzo anclado químicamente puede fluir antes de fallar por anclaje [5], surge la necesidad de hacer nuevos ensayos de flexión. Para ello, es suficiente ensayar una sola columna representativa de un parapeto, considerando dos situaciones: con anclaje químico y con anclaje natural de las barras verticales. Esta columna estaría sujeta a una carga horizontal monotónicamente creciente aplicada en su extremo superior.

\section{Agradecimiento}

Los autores desean expresar su agradecimiento a los estudiantes de la PUCP Karen Loo y Helmo Rodas, por su colaboración prestada en la primera parte del proyecto.

\section{Referencias}

[1] Ministerio de Vivienda, Construcción y Saneamiento y SENCiCo, "Albañilería”, Norma Técnica de Edificaciones E. 070, Reglamento Nacional de Edificaciones, Lima, 2006.

[2] The Euclid Chemical Company, "Euco \# 452 Epoxy System”, Claveland, OH, s. f. [En línea]. Disponible en: http://www.tamms.com/fileshare/ProductFiles/Spanish/SPA452SysLV.pdf

[3] Ministerio de Vivienda, Construcción y Saneamiento y sencico, "Diseño Sismorresistente", Norma Técnica de Edificaciones E.030, Reglamento Nacional de Edificaciones, Lima, 2006.

[4] Ministerio de Vivienda, Construcción y Saneamiento y sEncico. "Concreto Armado", Norma Técnica de Edificaciones E.060, Reglamento Nacional de Edificaciones, Lima, 2009.

[5] Á. San Bartolomé, A. Icochea, y A. Fernández, “Comportamiento de alféizares construidos con la Placa P-10 ante cargas sísmicas perpendiculares a su plano", Pontificia Universidad Católica del Perú, Lima, 2013. [En línea]. Disponible: http://blog.pucp.edu.pe/ media/688/20130515-articulo_icochea-fernandez.pdf 